Historic, Archive Document

Do not assume content reflects current scientific knowledge, policies, or practices. 



\section{GLAD BULBALOG}

Fall of 1928

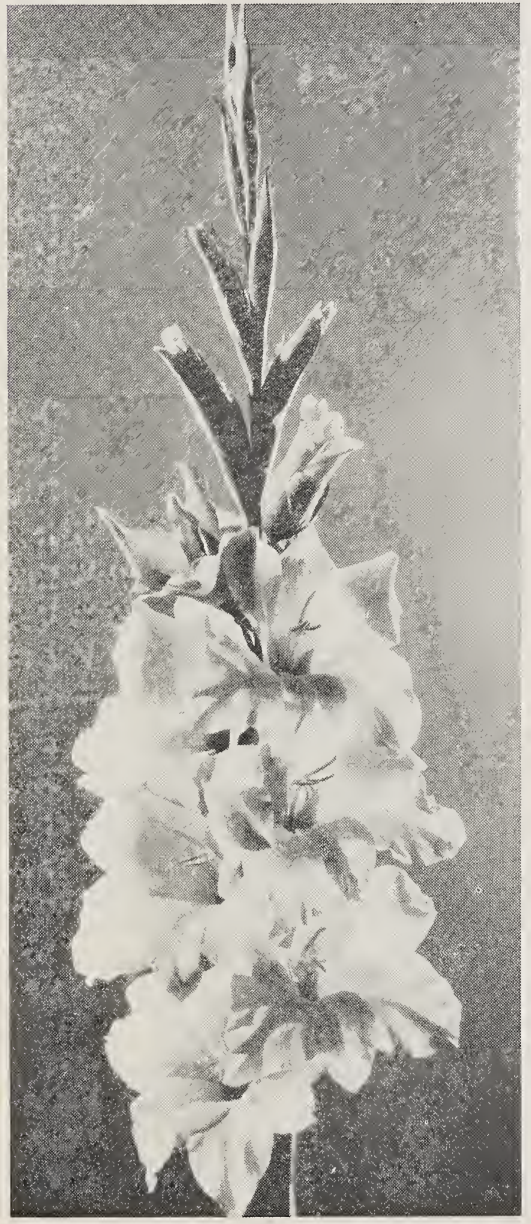

"Oregon Queen"

"Smiling Thru"

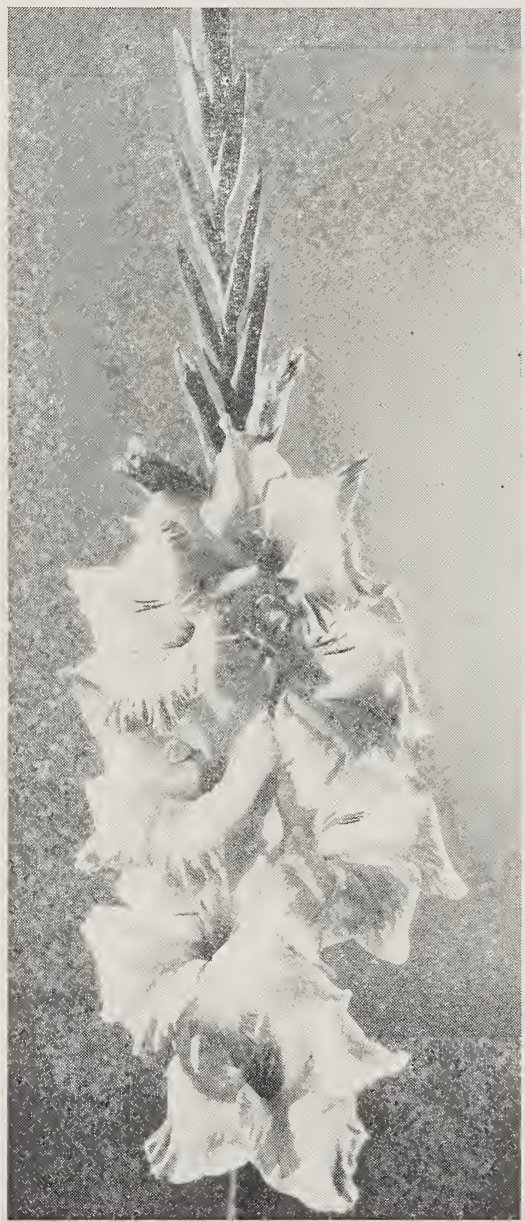

MERTON G. ELLIS BULB GARDENS

CANBY, OREGON 
Prices on this page are for No. 1 bulbs per each. For other sizes and bulblets, see next page.

ALVINA--A flesh pink of very dainty and ex. quisite coloring. $\$ 5.60$

ANGEL'S DREAM-La France pink of raie heauty. Many florets open at a time and rassy inare to open. Fine in basket and vase. ......... \$10.00

BF.ATRICIA-Soft rose-red with large wide-open florets. $\ldots 20 \mathrm{e}$

BENGAL BEALTY-A cros, between Pengal Figer and Golden Mcasure. Sitike is tall and strons like Golden Measue, but more the solor of Bengal Tiger, only more gold and brilliant markings. Dies not fade. It attracts much at. tention.

$\$ 2.50$

BRONZE MAID-The best we have ret seen in the bronze type. Beaut:ful blue thruat bloich. Strong and vigsrous. Very new and stock limited.

$\$ 25.00$

CAPT. COURACLOL'S-A large mutiled red of very rich ccluring and good form. ............ $\$ 2.50$

DOROTHY WOCl)-Very early. An improved and deeper pink than Prince of Wales. Highly recommended by Mr. T. H. Wuod of Canada, and named by him.

$\$ 3.10$

EARLY PHIPPS-An extra fine new arly varietv, a shade deeper in coloring than Mr. W. If. Phipps, one of its parents, but of the same shimmering, iridescent effect under electrie light where it is at its best in basket or vase. Slightly ruffled. Very early and of very last. ing quality. The best early one we bave

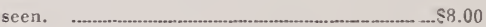

ELLA V. BIRNEY-A dainty pink of fine form and perfect placement of florets. ............. $\$ 5.00$

ELOMA- Winner of first prize for best new secd. ling at Northwest Regional Exhibit in i 1527. A Longfellow pink .............................. $\$ 1.00$

ERNESTINE-Very clear, bright, peach red with pure white throat. Sparkles in the sumlight or under electric light. Large florets and many out at once. Very beautiful. ................... $\$ 1.00$

FROST KING-A clear, coral pink with white throst. Graceful spike. Blooms very enrly and late. Early frests do not seem to affect it. Very prolific and splendid germinator. -.......2

CENERAL CANBY-Brilliant mottled red with beautifully feathered yellow throat. ........ $\$ 10.00$

GLoRIOLA-Peach red with yellow throat. A giant in the Grandiflora class.

$\$ 5.00$

GREY KNIGHT-A very odd but beautiful lavey. der gray. Its coloring reminds us uf $2 \mathrm{n}$ Iris.

$\$ 2.00$

LADY MACBETH-Large scarlet florets with two large white blotches in throat with thin dark red line running through center of eack. Many florets open. Good spike. Very strik. ing. $\$ 2.00$
LADY LORENE-Deep pink with yellew throat. Tall slender spike. Dainty and beantiful $\$ 3.00$

MISS CANBY-A dainty ruffled white with cream throat. This works up beautifully with Minuet or Mountain Girl in floral pieces. ............ $\$ 3.00$

MGNARCH OF TIE GLEN-The strongest growingglad in the garden. Immense spike. Extremely ruffled or fluted. bright salmon rink florets. In a class all by itself.

$\$ 20.00$

MOLNTAIN GIRL-A lavender pink about the culor of a w:ld rose, beautifully shaded with deeper pink and w:th a yellow throat. A ta!! sträght spike. Slightly ruffled. Miny florets open. A good heeper. Fine in floral

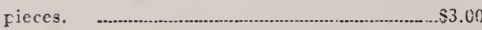

OIEGON QUEEN-Clear cream with yellow throat. Tall graceful spike. Large wide open florets, seven or more open at a time. Resembles, lovely lilies. $\$ 1.00$

rINK MARIE-An exccllent florists' piik. In form it somewhat resembles Varie Kun. derd. $.25 c$

PRIDE OF PORTLAND-Begonia rose. Very large florets. Good for exhibition use. W'inner of A. (. S. Trophy at Northwest Regional Exhibit in 1926 for best new seedling. ............ $\$ 5.00$

POINT TIIAIN-Soft pink, with pointed petals. Extra large florets on a large spike. Attracts much favorable comment. Fine for exhi. bition.

$\$ 15.00$

RED GLORY-A vigorous bright ruffled red of extra fine form and value. Except in color it closely resembles Purple Glory.

$\$ 200$

KED MARIE-Large red florets rnd iointed petals.

RID ROY-The tallest red. Large, weli plared florets. A very striking garden variety. $\$ 5.60$

RED TOR.NADO-Strong growing, irrilliant red with darker velvety throat. Heavy trxture. "It takes the world by storm, but storms don't tahe it."

sy. 00

K!l'FLIN, WATERS-Cream, suffuseil with rink. Large. Very beautiful in the rase. ............5.00

SNILING TIlRU-Ruffled lavender :renm. (None for sale this year.)

STAR OF IIIE SEA-Peach red with brownish scarlet throat tipped with yellow points. $\Lambda$ very beautiful glad which has attracted r:uci favorable comment. It is well named, "star of the Sea."

SIIORI) (OF NAIIOMET-An unusual shade of purpliah, brownish red. A strong grower. Very tall straight spike with well placed florets. An interesting oddity. $\$ 1.00$

WHITE CLOLD-A large creamy white, with shimmering effect under electric light. Many florets open. A good keeper, especially in late fall. 8100 


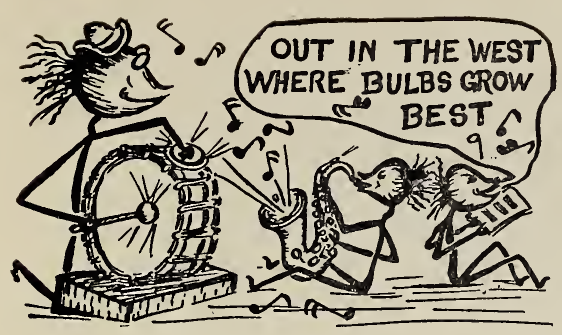

Bulbs

Per

A. B. Kunderd (K)

10

A. E. Kunderd (K)

Albatros (Pf.).

$1 \quad 1.75$

1

Albert 1st (V).......................... 1

Allen Bunce (Bill)..

Alma Rubens (D).

Altamyra (D).

Large Lilac.

Alton (K).

10

3.50

.50

2.25

.75

Alvina (E)

1
10

100

Angel's Drean

Anna Eberius (D)................... 10

10

Popular Velvety Purple......... 100

Balboa (Br.)................................. 1 Apricot Pink.

10

Barbara Lamar (D)................... 1

Beatricia (E)................................ 10

Soft Red...

100

Beatriz Michelena (D)............ 10

Peach Red Orange................. 100

Bengal Beauty (Mrs. E.)........ 1

Bengal Tiger (P).................... 10

Brilliant mottled red............... 100

Bernard Shaw (D).................... 10 Lilac-tinted............................... 100

Betty Joy (Bill)........................ 10

Betty Nuthall (S)...................... 1

Blanche Bollinger (K)............. 10

Bobby (K) ............................... 10

Rose, with deep blotches..... 100

Bon Echo (Bon.)......................... 1

Break o' Day (Bill).................. 10

Very early pink.......................... 100

Byron L. Smith (K)................. 10 Lavender.................................... 100

Canandaigua Red (Bill)............ 10

Capt. Courageous (E).............. 1

Cardinal Prince (K).................. 10

Tall, Cardinal............................. 100

Carmen Sylva (Dec.)................ 10 White.

100

Catalina (Br.).

Rose Pink..

10

Catherine Coleman (C)............ 10

Chalice Flower (K).

.80

5.00

1.00

7.00

1.50

1.00

8.00

1.75

15.00

.50

4.00

1.75

14.25

7.50

.50
1.00

8.00

4.00

2.90

24.00
Our bulbs are grown on a fine sandy loam, in Oregon, the home of fine bulbs. ..We/ use NEW soil each year. The bulbs are carefully cultivated, irrigated, and finally, when dug, they are cured and placed in storage in shallow trays with fly screen bottoms, in our commodious frost-proof bulb house, where they await your orders.
No. 1 No. 2 No. 3 No. 4 No. 5 No. 6 Bulblets

11/2-up 11/4-11/2 1-11/4 3/4-1 1/2-3/4 $1 / 2$-Less Per

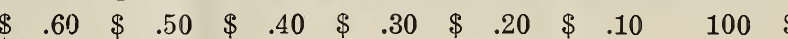

.90

1.50

1.15

.85

.60

.40

10

$\$ \quad .20$

3.00

.45

2.00

.65

.90

.70

8.50

.50

.40

.85

6.00

1.25

.85

6.50

1.50

12.00

$\begin{array}{ll}2.50 & 2.00\end{array}$

.40

3.00

1.50

11.50

2.75

6.75

2.25

.45

6.75

.40

1.75

2.50

20.00

16.00

3.20
.40

1.75

5.70

1.50

1.25

.35

.35

5.75

.50
4.50

3.00

.50

.60

3.50

3.00

4.50

3.50

6.00

.25

.35

2.00

.15

1.00

.70

5.00

.55

4.00

.40
3.25

1.00

.80

.70
5.25

.50

3.50

1.00

.75

9.00

6.25

3.25

.60

.30

2.25

.50

4.50

.35

2.50

.25

2.00

.15

1.25

9.00

.90

7.00

.65

2.35

1.80

6.00

5.00

2.00

1.75

.90

.70

6.00

.40

.35

.65

.55

5.75

4.50

.35

.25

2.50

2.00

1.50

1.25

2.00

1.75

2.00

1.50

12.25

25.

.35
250

2.00

2.50
70

.55

5.00

4.00

1.85

1.60

1.25

$\begin{array}{rrr} & 100 & 3.00 \\ 1.00 & 1000 & 6.00 \\ .25 & 1 & .10 \\ .25 & 100 & .25 \\ 2.00 & 1000 & 2.00 \\ & 100 & .25\end{array}$

2.50

2.75

.10

.80

.25

2.25

.40

.20

1.50

.40

$3.25^{\circ}$

10

4.00

10

7.50

100

.10

1000

100

1000

10

3.00

27.00

1.25
.20

100

.20

100

1000

10

$.10 \quad 100$

$.80 \quad 1000$

$.50 \quad 100$

$4.25 \quad 1000$

5.50

1.50

1.00

1.50

1.25

.50

4.00

.30

.40

3.00

.15

1.00

.90

1.50

1.00

9.00

15.

1.00

.40

3.25

1.25

1.50

1.00

.25
.35

2.80

.20

.20

1.50

.10

.80

.50

1.25

.70

6.25

.10 


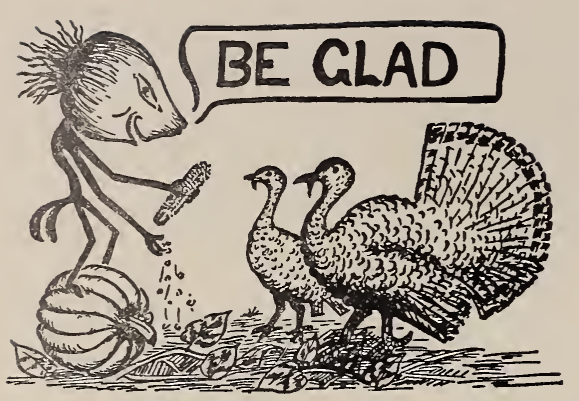

Bults

Per

Chas. F. Fairbanks (K)........... 10 Red.......................................... 100

Chiffonette $(K)$.......................... 10

Col. Lindbergh (H)................. 1

Copper Bronze (K)................... 10

Coronado (Br.)......................... 1

Cowee's Scarlet Wonder............ 10

Large Glowing Red.................. 100

Creatore (Bill)............................ 1

Crimson Glow (Bet.)................ 10

Bright Glowing Crimson...... 100

Crinkles (K) ............................ 10

Diana (J. Z.) ............................. 10

Diener's American Beauty........ 10

American Beauty Rose.......... 100

Dorothy Wood (Mrs. E.).

Dorrit (K). Lavender Pink

10

Dr. Crist Martz. (K).

Ruffled Red..

100

Dr. F. E. Bennett (D) .............. 10

"House Afire" Red................ 100

Dr. Hans Pfitzer (Pf.)............. 1

Dr. J H. Neeley (K) .................. 10

Blushed White......................... 100

Dr. W. Van Fleet (K).............. 10

Early Rose Pink ................... 100

Early Phipps (E)...................... 1

E. J. Shay'or (K) ..................... 10

Ruffled Rose Pink.................... 100

Eldora (Kemp) ........................... 10

Buff P'ink............................... 100

Elf (D) ................................... 10

Fine Florists' White............... 100

Elizabeth Tabor (Hinkle)........ 10

Early Blotched Pink.............. 100

Ella V. Birney (E).................... 1

Eloma (E)................................. 1

Elora (Bur.) .............................. 10

Cream White.......................... 100

Ernestine (Mrs. E.).................. 1

Ethelyn (Fis.).......................... 10

Amber Yellow..

100

Eula Terry (Bon.)

1

Evelyn Kirtland (A)

Tall Pink
No. 1 No. 2 No. 3 No. 4 No. 5 No. 6 Bulblets

11/2-up 11/4-11/2 1-1 1/4 3/4-1 1/2-3/4 1/2-Less Per

190

8.20

.80

.65

2.00

6.50

5.25

4.00

1.70

1.35

2.50

3.50

2.25

2.10

1.75

10.00

.55

4.50

9.00

8.00

.45

.35

3.00

.50

4.00

3.50

2.50

2.50

2.00

.40

.35

1.50

3.20

2.50

1.25

1.00

.50

4.25

.40

$.30 \quad 100$

1.00
1.75

3.00

2.00

1000

.15

.75

.40

100

1.00

1.50

1.25

1.2i)

1.35

1.00

.65

7.00

.25

1.50

.15

1.00

1.75

1.50

.25

2.00

.15

1.00

.65

.85

.35

.65

5.50

.55

.45

.25

.15

.35

.20

2.50

1.20

(See Specia

.50

4.00

.35
250

Sets on

Next to

.15
1.00

2.00

.40

3.20

.50
.30

.25

3.00

.35

2.50

2.25

16.00

.75

1.40

1.00

12.00

8.40

.75

.65

.55

6.00

.45

.50

.40

.30

.25

4.00

3.00

2.25

1.75

.50

.40

.35

.25

2.50

2.00

7.50

.50

4.00

6.25

5.00

4.00

.35

.40

2.50

.25

2.00

1.40

16.75

12.25

1.00

.50

4.00

.40

8.40

.75

.35

3.20

2.50

.25

.50

.40

.35

2.50

2.00

.25

2.00

20

1.75

.50

4.25

.35

3.50

4.00

.70

1.00

.90

.80

.25

4.00

.40

.35

1.00

3.20

2.50

2.00

.80

1.15

.90

1.00

8.50

.85

.70

.65

7.00

5.50

.50

.45

.40

.35

.50

.40

.35

.25

2.50
.15

1.00

3.00

.15

1.00

.50

4.00

.15

1.00

.15

1.00

3.00

.60

.15

1.00

.50

.50

4.00

.30

.15

1.00$$
10
$$$$
.10
$$

1.25

.10

.80

.50

.10

$.10 \quad 100$

$.80 \quad 1000$

2.50

.25

2.00

.10

.80

3.60

100

1000

10

.10

.80 
If you would win success in the growing of bulbs, you must have bulbs of highest quality and of the hest varieties. Otherwise, you have about as much chance of winning as would a poor cripple on a crutch in a Marathon race. So why grow the poor and the cheap when the best often costs but very little more?

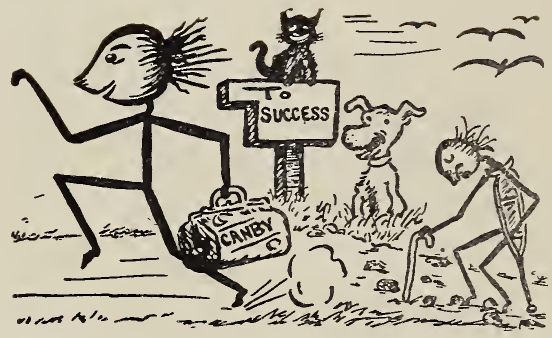

Bulbs

Per

Fallen Leaf (M) ...................... 10

Fastidious (Bill) .................... 10 Shell pink .............................. 100

Fern Kyle (K) .......................... 10

Ruffled Creamy White.......... 100

Fontaine (C) ........................... 10 Dainty Pink.............................. 100

Frank M. Shick (S) .................. 1

Frost King (Mrs. E.)............. 10 Coral Pink............................. 100

Gay Hussar (S)...

Genersl Canby (E)................... 1

Gene Stratton Porter (K)....... 10 Friiled Pink .......................... 100

Geo. J. Joerg (J) .................... 1 Deep Clear Pink..................... 10

Geraldine Farrar (D)............... 1 Light blue.............................. 10

Gertrude Errey (Er.)............... 10 Bright Shell Pink.................... 100

Giant Myrtle (K) ....................... 10

Giant Nymph (C) ...................... 10

Gladdie Boy (Bill)................... 10

Rose Pink............................... 100

Gladis Plath (D)....................... 10

Mallow Purple.......................... 100

Gladscme (Suttle)..................... 1

Glendale (Davis)....................... 10 Tall Purple................................. 100

Gloriana (Bet.)........................ 10 Fine salmon, yellow throat 100

Gloriola (E) ............................. 1

Gold Eagle (A) .......................... 10

Early Yellow........................... 100

Golden Dream (Gr.).................. 10

Tall Deep Yellow.................... 100

Golden Frills (K) ...................... 10

Deep Ruffled Yellow............. 100

Golden Measure (Kel.)............. 10 Large Tall Yellow.................... 100

Golden Salmon (K) ..................... 10

Buff Salmon............................. 100

Golden Treasure (Bar.)............ 1.

Grey Knight (E) ....................... 1

Halcy on (Bili) .............................. 10

Hawaii (D)................................. 10

Heavenly BIue (Pf.).

No. 1 No. 2 No. 3 No. 4 No. 5 No. 6

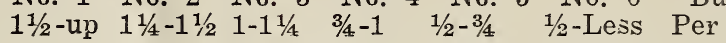

$\begin{array}{lll}5.00 & 4.50 & 4.00\end{array}$

1.00

9.00

.85

.75

3.50

2.75

2.00

100

Bulblets

.80

7.75

6.50

.65

7.00

.65

.55

.50

4.00

5.75

4.50

5.25

.45

3.50

.35

5.00

3. 20

2.50

.25

2.00

1.50

4.50

4.00

3.50

12.00

1.25

1.10

5.00

11.00

9.55

.90

8.00

10.00

4.50

4.00

3.50

.90

8.00

9.00

8.00

7.00

.70

7.00

1.00

8.50

.85

5.75

.60

4.50

.75

.65

.50

4.50

7.50

6.50

5.50

.40

4.00

1.25

10.00

1.00

1.75

8.75

3.50

.35

2.75

.70

7.50

6.25

.75

.50

1.00

.80

.60

.45

1.50

1.25

1.10

12.50

11.00

.50

.40

9.50

.90

8.00

.25

.35
250

2.00

.35

.30

25

.50

4.00

4.00

36.00

5.00

6.00

50.00

8.00

70.00

6.00

.40

.35

3.20

2.50

3.00

30.00

24.00

4.25

3.50

5.00

40.00

7.00

4.00

30.00

6.00

60.00

50.00

5.00

4.00

.80

7.00

.70

.60

4.00

30.00

6.00

5.00

3.00

1.50

22.00

2.25

16.00

1.00

1.25

1.50

2.00

1.75

2.00

2.50

.30
6.00

.60

.50

800

7.00

6.00

.25

2.00

2.25

18.25

3.00

3.00

20.00

5.00

40.25

.50

4.00

.35

2.50

.15

1.00

.40

3.00

100

.25100

$1.50 \quad 1000$

$.10 \quad 100$

$.80 \quad 1000$

10

.75

6.55

60

5,00

100

1000

10

6.00

.45

3.00

.55

4.50

.25

2.00

.55

4.75

.35

.35

75

6.50

.15

1.00

.20

.15

1.00

1.50

12.25

2.50

2.00

12.25

4.00

30.25

3.00

20.00

1.75

.50

4.00

12.00

1.50

10.25

.80

1.25

1.65

.20

5.25

.35
2.75

.75

6.25

.65

1.00

1.30

4.25

.70

.90

.80

1.50 


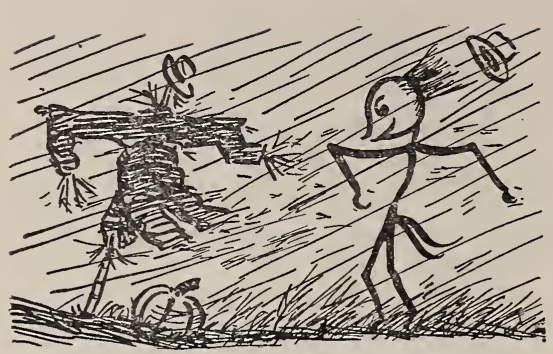

\section{STRAWS SHOW WHERE BIG WINDS BLOW}

A good bloom is a mighty good indication of a good bulb at the bottom of the spike. Our bulbs are full of pep and produce prize-winning exhibition blcoms both for us and for the many who have bought from us and who report fine success.
Bulbs

Per

H. G. Wells (D)

Purplish Red.

1

Henry Ford (D)

Deep Purple.

Herada (A) Magenta.

Herbstzauber (Pf.).

Highland Laddie (K). Strangely marked Pink 100

Hope Hampton (D)................. 1

Imp'd Queen of the Night (D) 10 Deep Maroon........................... 100

J. A. Carbone (D).................. 10 Iridescent Orange ................ 100

Jack London (D)....................... 10 Orange Salmon....................... 100

Jacoba van Beijeren (Eu.)........ 10 Phlox Purple........................... 100

Jean du Tailles (Eu.)................ 1

Jean Tenny (Nic.)...................... 10 Lavender Pink 100

Jenny Lind (Dec.).................... 10 Dainty Pink and Cream ........100

Jewell (C. Z.) ........................... 10 Pink with yellow throat ........100

Joerg's White.............................. 1 Very Large White................. 10

J. Ogden Armour (K) ............. 10

John D Oliver (K).................... 1 Deep Lavender.......................... 10

John Dunbar (K) ..................... 10

John T. McCutcheon (K).......... 1 Red......................................... 10

John T. Pirie (K).................... 10 Very Striking Oddity............. 100

Juniata, (Kemp). Pink with white throat......... 10

Karl Volkert (Pf.)................... 10

Katharine F. Hess (D)............ 10 Tall Deen Pink....................... 100

Kitty Grullemans (Eu.)............ 10 Pink and Cream................... 100

Kunderd's Yellow Wonder........ 10 Vigorous Yellow..................... 100

Lacinatus (K) ............................ 10

Jady Primera (H)................... 1

Lady MacBeth (Mrs. E.)........ 1

Lilac Wonder (Eu.)......................10 Lavender Lilac.......................... 100

No. 1 No. 2 No. 3 No. 4 No. 5 No. 6

$1 \frac{1 / 2}{1}$ up $1 \frac{1 / 4}{1}-1^{1 / 2} \quad 1-1^{1 / 4} \quad 3 / 4-1$

$1 / 2-3 / 4$
$1 / 2$-Less

Bulblets

$\begin{array}{llll}1.00 & .80 & .60 & .45\end{array}$

$\begin{array}{lll}9.00 & 7.25 & .60\end{array}$

.50

4.00

.40

3.20

.35

.50

4.00

.40

3.20

2.50

.25
2.00

.35

2.50

.25

.50

.45

.40

2.00

.35

.80

.70

$\begin{array}{ll}7.00 & 6.00\end{array}$

.60

.50

1.00

.85

3.50

3.00

5.00

4.00

.60

2.50

2.00

22.50

18.00

.65

6.00

.55

.45

.35

.50

4.00

4.80

3.60

2.80

.35

.25

3.20

.75

6.50

.65

5.50

2.50

2.00

.55
4.50

.40

3.50

.40

.35

1.75

15.00

.45

1.25

1.00

13.00

11.00

9.00

.50

$6.00 \quad 5.00$

4.00

.40

.50

.35

3.00

.25

2.50

2.00

$4.00 \quad 3.20$

$.60 \quad .50$

5.00

4.50

.45

4.00

.35

3.50

.50

4.00

3.00

2.50

3.25

.45

3.50

.40

2.00

3.00

.30

2.50

.35

3.15

.25
-2.25

.15

1.00

.10

100

.75

.15

1.00

.30

.40

3.25

.40

1.50

13.50

.25

1.90

.15

1.00

.30

2.00

.80

100

1000

$.10 \quad 100$

$.80 \quad 1000$

.25

10

$.30 \quad 100$

2.25

1000

.35

10

1.00

9.00

100

.15

1000

$1.15 \quad 100$

$.10 \quad 100$

$.80 \quad 1000$

$.15 \quad 100$

$1.00 \quad 1000$

10

.80

7.25

.60

5.25

100

1000

.30

2.00

.15

1.25

100

1000

.15

.10

100

1.00

.30

2.50

.80

1000

.20
1.75

10
100

1.50

.25

2.00

1.00

.10
150

.50

.65

.55

.45

.40

4.00

3.50

1.50

13.00

1.25

3.00

.35

2.50

.30

2.00

.80

.60

9.00

7.00

5.25

11.00

.35

3.50

.30

.25

3.50

.60

5.25

2.50

2.00

1.50

2.50

.50

.50
4.50

.45

2.00

1.50

.40

3.75

3.00

.30
2.00

.35

4.00

.40

2.50

.25

2.00

2.00

1.00

4.50

2.75

5000

40.00 


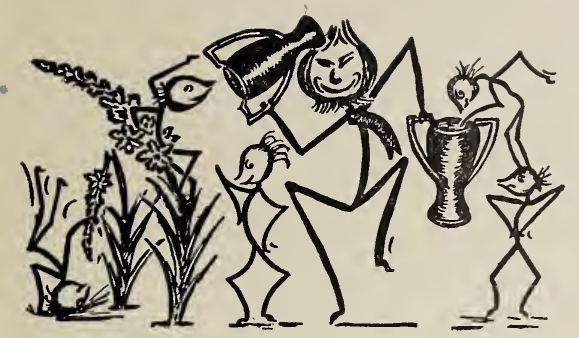

CUPS SHOW WHERE GOOD BULBS GKUW

Grand-Sweepstakes Gold Cup for most poin t s, Sweepstakes Silver Cup for Best Spike, First for Best Exhibit, First for Best New Seedling, Etc., were won by us at the Northwest Regional Glad Show, both in 1927 and in 1928.

\section{Per}

Longfellow (Dec.) Deep Pink................................ 100

Lorice (Kemp)........................ 10 Ruffled Scarlet Red............... 100

Los Angeles (Houd.)................ 10 "Cut and Come" Pink.......... 100

Mabel Irving (Br.).................... 1

Madison Cooper (K)................ 10 Purplish Satiny Red.............. 100

Marie Kunderd (K)................. 10 Fine Early White.................... 100

Marietta (M).............................. 10 Extra Fine Salmon................. 100

Marnia (Kemp) .......................... 10 Grenadine Orange.................. 100

Marshal Foch (K)................... 10 Beautiful Salmon Pink........ 100

Mary Pickford (K)................... 10 Fine Florists' White............... 100

Masterpiece (K) ...................... 10 Ruffled American Beauty.... 100

Merced (Br.) .............................. 10 Orange Saffron......................... 100

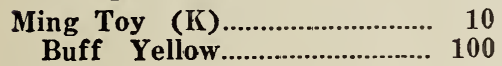

Minuet (C)................................. 1 Most Beautiful Lavender...... 10 Great Prize Winner............... 100

Miss Canby (E)...

Mission Bells (Br.)................... 1 Orange Pink............................... 10

Miss Madison (Boy.)............... 10 Large Light Pink....................... 100

Miss Spokane (K).................... 10 Ruffled Orange Salmon........ 100

Mixture, many kinids ................ 100

Monarch of the Glen (Mrs. E.) 1

Mountain Girl (Mrs. E.).......... 1

Mrs. Dr. Norton (K) ............... 10

Mrs. Ella G. Morrison (Kir.).... 1

Mrs. F.. C. Hornberger (Hor.) 1 Fine New White................... 10

Mrs. F. C. Peters (Fis.) .......... 10 Tall Lavender........................ 100

Mrs H. E. Bothin (D)............... 10 Creamy Frilled Pink............... 100 Mrs. J. C. Bruggen (K) ........... 10
Rose Pink ............................... 100
No. 1 No. 2 No. 3 No. 4 No. 5

$11 / 2$-up $1 \frac{1}{4}-1 \frac{1}{2} \quad 1-1 \frac{1 / 4}{3 / 4}-1 \quad 1 / 2-3 / 4$

2.25

20.00

1.80

16.50

2.00

16.00

1.50

12.00

1.60

.70

6.00

14.50

1.40

12.50

1.00

.50

4.00

.70

6.00

1.10

9.00

1.25

10.00

.70

6.00

.55

4.75

.65

5.25

5.50

.50

4.00

1.25

11.10

100.00

2.00

.65

5:50

.80

7.00

.65

5.50

2.25

20.00

3.00

.50

.50

1.00

9.00

.65

560

.50

4.00

1.50

.60

5.00

.50
4.00

1.00

8.50

1.20

10.25

.35

2.75

.75
6.25
1.00
8.00
.25
1.65

.40

3.20

.55

4.75

1.00

8.00

1.00

8.25

.55

4.75

.45

4.00

.55

4.50

4.50

.40

3.20

.35

2.50

.45

3.60

.90

7.20

.75

6.50

.45

3.60

.40

3.25

.45

3.75

3.50

.35

2.50

1.05

9.40

84.25

1.75

.55

4.50

.70

6.00

.55

4.50

.90

8.20

72.25

1.50

.45

3.50

22.00

60.25

.60

5.00

.45

3.50

$1.90 \quad 1.55$

2.70

2.35

.45

.40

.45
.85

7.65

.40

.70

6.30

.55

4.80

.45

3.60

.40

3.20

.35

1.25

2.50
1.00

10.50

8.25
.25

2.00

.30

2.20

.75

6.30

.55

4.75

.30

2.25

.30

2.25

.35

3.00

2.50

.25

2.00

.75

6.75

1.25

.35

2.50

.50

4.00

.35

2.50

1.10

2.00

.30

.35

\section{.15}

1.00

.20

1.40

.60

5.40

.45

3.75

.20

1.40

.20

1.25

.25

2.00

1.75

16.00

.15

1.00

.55

4.80

44.25

1.00

.25

1.60

.35

2.45

$.2 \mathrm{~b}$

1.50

.80

8.00

1.50

.20

.30

.35

3.15

.35

.25

2.40

1.60

.25
2.00

.15

.80

1.00
.60

7.00

No. 6 $1 / 2$-Less Per $\begin{array}{rrr}.50 & 100 & 1.00 \\ 4.25 & 1000 & 8.00 \\ .80 & 100 & 1.60\end{array}$

$\begin{array}{rrr}6.00 & 1000 & 14.25\end{array}$

$\begin{array}{lll}.15 & 100 & .20\end{array}$

$\begin{array}{lll}.90 & 1000 & 1.40\end{array}$

$10 \quad 1.00$

$\begin{array}{rrr}.10 & 100 \quad .10\end{array}$

$\begin{array}{lll}.10 & 100 \quad .10\end{array}$

$\begin{array}{lll}.80 & 1000 & .80\end{array}$

$\begin{array}{lll}.45 & 100 \quad .25\end{array}$

$\begin{array}{lll}3.75 & 1000 & 2.15\end{array}$

$\begin{array}{lll}.35 & 100 & .50\end{array}$

$\begin{array}{lll}2.50 & 1000 & 4.00\end{array}$

$\begin{array}{lll}.10 & 100 \quad .20\end{array}$ 


$\begin{array}{lr}\text { Altamyra .................. } & \$ 5.00 \\ \text { Anna Eberius ........... } & 3.00 \\ \text { Beatricia ................... } & 5.00 \\ \text { Bengal Tiger .............. } & 3.00 \\ \text { Break O'Day ............. } & 9.00 \\ \text { Byron L. Smith ............ } & 3.00 \\ \text { Carmen Sylva .......... } & 3.00 \\ \text { C's Scarlet Wonder .... } & 3.50 \\ \text { Crimson Glow ......... } & 3.00 \\ \text { Dorrit ................... } & 3.00 \\ \text { Dr. F. E. Bennett ...... } & 12.00\end{array}$

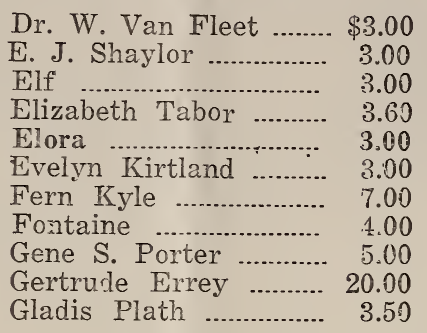

Glendale

Gloriana

Henry Ford

Herala

J. A. Carbone

Jack London

Jacoba V. Beijeren .... 4.50

Je:nny Lind ................ 5.50

Jewell ……....................... $\quad . .40$

Katharine F. Hess .... 4.00

Kitty Grullemans ...... 2.50
Builos

Per

Mrs. J. C. Grossman (G) .......... 10

Deep Salmon Primulinus 100

Mrs。 J. K. Armsby (D) ............ 10

Coral Pink ........................... 100

Mrs. John R. Walsh (D) ............ 10

Mrs. Leon Douglas (D) ............ 10

Very large Salmon Pink ...... 100

Mrs. Mary S. Burke (D) ............ 10

Apricot Yellow .................... 100

Mrs. P. W. Sisson (C) ................ 10

Ruffled Pink ........................ 100

Mrs. Robt. Cunningham (D)...... 10

Tall Lavender Pink ............. 100

Mrs. Richard Lohrman (ID) ...... 10

Creamy Shell Pink ............. 100

Mrs. T. Rattray (Australia) .... I

Tall Rose Pink ..................... 10

Mrs, Van Konynenburg (Eu.) ......1

Mrs. W. E. Clark (Fis.) ............ 10

Flesh Pink, Red Blotch ........ 100

Mr. W. H. Phipps (D) ............. 10

Iridescent Pink. Many Open 100

Nimrod (Australia) .................... 10

Nixie (D) ................................ 1

Opalescent (Bill) ...................... 10

Fine Florists' Lavender ......... 100

Orchid Lady (Sp.) ................... ..1

Oregon Queen (Mrs. E.) ........... 1

Othello.(Pf.) ............................. 1

Patricia Carter (K.) ............... 1

Paul Pfitzer (Pf.) …............... 1

Pearl of the Dawn (K) ........... 10

Very Ruffled Pink ................ 100

Pfitzer's Triumph ................... 1

Very Large Salmon Orange io

Phaenomen (Eu.) ..................... 1

Phini (D) ................................ 1

Pink I.ily (II) .............................. 10 Soft Purplish Pint .............. 100

Pink Marie (E) ...................... 10

Fine F!orists' Pink ................ 100

Pink Wonder (Kemp) .............. 10 Large Light Pink ..................... 100

Point Twain (E) ............................1

Pola Negri (D) ........................ 10 Apricot
No. 1 No. 2 No. 3 No.

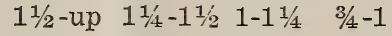

$\begin{array}{rrrr}.65 & .55 & .45 & .35 \\ 5.50 & 4.50 & 3.50 & 2.50\end{array}$

$5.50 \quad 4.50$

3.50

.75

6.75

.60

1.10

5.50

.45

.80

7.20

.95

4.00

.65

.65

6.00

.85

.55

5.75

.55

4.80

5.50

4.75

.45

50.00

4.50

3.75

3.50

1.60

14.50

40.00

30.00

1.45

.75

6.25

12.25

1.20

10.00

.65

5.25

.55

2.25

18.50

2.00

4.00

22.25

17.25

1.75

1.90

1.70

16.00

13.50

1.50

6.75

2.25

20.00

2.00

.75

.65

6.00

16.00

4.00

1.50

1.80

.90

.65

5.50

1.50

13.50

.35

.35

.70

6.00

11.25

.85

7.75

.50

4.00

10.00
.55

.65

4.25

1.75

1.25

12.25

1.75

1.50

.65

.55

.45

5.00

4.00

2.50

2.00

1.30

1.10

1.60

.80

.70

.55
4.75

.45

1.25

4.00
1.00

9.00

.30

.25

.30

.60

5.00

.45

3.90

.75

6.50

.40

.45

3.25

1.00

9.25

1.25

.45

.30

2.40

1.50

.90

1.20

.60

.35

3.00

.85

7.65

.20

.20

.35

2.90

.50

5.25

3.20

.35
2.50

4.00

.25

9.00

8.00

7.00
.30
2.75

.75

.40

3.60

.35

2.75

2.50

1.00

8.25

.40

2.75

1,50

1.30

No. 5

$$
1 / 2-3 / 4
$$

$$
\begin{array}{r}
.25 \\
1.75
\end{array}
$$

1.75

.20
1.75

.60

.30

2.40

.25

1.75

1.50

12.25

.80

6.25

.25

1.75

1.25

10.50

1.00

.40

.75

6.00

1.00

.35

.20

1.60

1.00

.35
2.75

No. 6 1/2-Less Per

$.15 \quad 100$

$1.00 \quad 1000$

$.10 \quad 100$

$.90 \quad 1000$

$.45 \quad 100$

$.20 \quad 100$

$1.60 \quad 1000$

$.15 \quad 100$

$1.00 \quad 1000$

$1.00 \quad 100$

8.25

.55

4.00

.15

.85

1.00

9.00

.70

1000

100

1000

100

1000

10

$100 \quad 2.00$

10

.35

100

1000 
Lorice

$\$ 40.00$

Los Angeles ................ $\quad 5.60$

Madison Cooper ........ $\quad 2.50$

Marnia ......................... 15.1)0

Marshal Foch (K) ... $\quad 4.00$

Mary Pickford ............ : ¿.00

Masterpiece ................ $\quad 6.00$

Minuet ........................ 250.00

Miss Madison ........... $\quad 7.00$

Miss Spokane ............. $\quad 4.00$

Mrs. F. C. Peters ...... 4.00
Mrs. H. E. Bothin ...... \$ 2.50

Mrs. J. C. Grossman .. $\$ .50$

Mrs. J. K. Armsby .... 4.50

Mrs. Leon Douglas .... $\quad 6.60$

Mrs. P. W. Sisson .... 90.00

Mrs. R. Lohrman ..... $\quad 3.50$

Mr. W. H. Phipps ..... 18.00

Opalescent .................. 4.00

Pearl of the Dawn .... $\quad 3.50$

Pink Lily .................. $\quad 4.00$

Pink Marie ................ 12.00
Pink Wonder

3.00

Purple Glory

7.00

Red Fire

Richard Diener

5.50

Rose Ash

stalwart

Princely

Tycko Zang.

Wm. G. Badger ........

White Wonder
8.00

3.00

5.50

9.00

7.00

\begin{tabular}{|c|c|c|c|c|c|c|c|c|c|}
\hline & $\begin{array}{l}\text { Bulbs } \\
\text { Per }\end{array}$ & $\begin{array}{l}\text { No. } \\
11 / 2-u\end{array}$ & $\begin{array}{ll}1 & \text { No. } \\
\text { Ip } & 11 / 4-\end{array}$ & $\begin{array}{ll}2 & \text { No. } 3 \\
11 / 2 & 1-1 \frac{1 / 4}{}\end{array}$ & $\underset{3 / 4-1}{N o}$ & $4 \underset{1 / 2-3 / 4}{\mathrm{No}}$ & $\begin{array}{r}\text { No. } 6 \\
1 / 2-\text { Less }\end{array}$ & $\begin{array}{l}\mathrm{Bu} \\
\mathrm{Per}\end{array}$ & \\
\hline President Harding (Par.)........ & 10 & 2.00 & 1.75 & 1.50 & 1.25 & 1.00 & .75 & 100 & 1.50 \\
\hline Pride of Portland (E) ................ & 1 & 5.00 & 4.25 & 3.50 & 2.75 & 2.00 & 1.00 & 10 & 3.50 \\
\hline $\begin{array}{l}\text { Primadonna (Fis.) } \\
\quad \text { Yellow, Primulinus }\end{array}$ & 100 & & & & $\begin{array}{r}.35 \\
2.75\end{array}$ & 1.75 & $\begin{array}{r}.15 \\
1.00\end{array}$ & $\begin{array}{r}100 \\
1000\end{array}$ & $\begin{array}{l}.10 \\
.90\end{array}$ \\
\hline Princely (Kemp) ...... & 10 & 1.00 & .85 & .70 & .55 & .40 & .25 & 100 & .25 \\
\hline Extra Fine White & 100 & 9.00 & 7.50 & 6.00 & 4.50 & 3.00 & 1.50 & 1000 & 2.25 \\
\hline $\begin{array}{l}\text { Prince of Wales (V. Z.) } \\
\text { Early Salmon }\end{array}$ & $\begin{array}{r}10 \\
100\end{array}$ & $\begin{array}{r}.50 \\
4.00\end{array}$ & $\begin{array}{r}.40 \\
3.20\end{array}$ & $\begin{array}{r}.35 \\
2.50\end{array}$ & $\begin{array}{r}.25 \\
2.00\end{array}$ & $\begin{array}{r}.15 \\
1.00\end{array}$ & $\begin{array}{l}.10 \\
.80\end{array}$ & $\begin{array}{r}100 \\
1000\end{array}$ & $\begin{array}{l}.10 \\
.80\end{array}$ \\
\hline Priority (Bill) & ... 10 & 1.00 & .80 & .60 & .45 & .35 & .25 & 100 &.$\overline{0} 0$ \\
\hline Purest of Al! (Eu.) & 1 & .75 & .65 & .55 & .45 & .35 & .25 & 10 & .50 \\
\hline Purple Glory (K). & ... 10 & .80 & .65 & .55 & .45 & .35 & .25 & 100 & .25 \\
\hline Ruffled Purplish Red & -100 & 7.00 & 5.75 & 4.40 & 3.20 & 2.40 & 1.60 & 1000 & 2.00 \\
\hline $\begin{array}{c}\text { Quinton (Kemp) } \\
\text { Coral Pinls }\end{array}$ & $\begin{array}{r}10 \\
-100\end{array}$ & & & & .25 & .15 & .10 & 100 & .10 \\
\hline Red Fire (K) & 10 & .80 & .65 & .55 & $\begin{array}{r}1.90 \\
.45\end{array}$ & $\begin{array}{r}1.25 \\
.30\end{array}$ & .75 & $\begin{array}{r}1000 \\
100\end{array}$ & .15 \\
\hline Very Red. Stands heat & 100 & 7.00 & 5.75 & 4.50 & 3.25 & 2.25 & 1.25 & 1000 & 1.25 \\
\hline Red Glory (E) & 1 & 2.00 & 1.75 & 1.50 & 1.25 & 1.00 & .75 & 10 & 1.50 \\
\hline Red Roy (E) & 1 & 5.00 & 4.50 & 3.75 & 3.00 & 2.50 & 2.00 & 10 & 3.50 \\
\hline Red Tornado (E) & 1 & 9.00 & 8.00 & 6.85 & 5.50 & 4.25 & 3.00 & 10 & 6.00 \\
\hline Richard Diener (D) & 10 & .70 & .55 & .45 & .35 & .25 & .15 & 100 & .25 \\
\hline Famous Pink ........ & 100 & 6.00 & 4.75 & 3.80 & 2.80 & 2.00 & 1.20 & 1000 & 1.40 \\
\hline Rippling Waters (E) & 1 & 5.00 & 4.50 & 3.75 & 3.00 & 2.50 & 2.00 & 10 & 3.50 \\
\hline Rita Beck (Fis.) & 1 & 2.00 & 1.60 & 1.15 & .90 & .70 & .50 & 10 & 1.15 \\
\hline 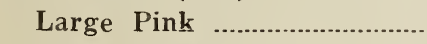 & 10 & 15.00 & 13.00 & 10.50 & 8.50 & 6.25 & 4.50 & 100 & 10.00 \\
\hline $\begin{array}{l}\text { Rebt. J. Kunderd (K) } \\
\quad \text { Fine Red }\end{array}$ & $\begin{array}{r}10 \\
100\end{array}$ & $\begin{array}{r}.95 \\
8.40\end{array}$ & $\begin{array}{r}.80 \\
7.20\end{array}$ & $\begin{array}{r}.65 \\
5.60\end{array}$ & $\begin{array}{r}.50 \\
4.00\end{array}$ & $\begin{array}{r}.40 \\
3.00\end{array}$ & $\begin{array}{r}.25 \\
1.80\end{array}$ & $\begin{array}{r}100 \\
1000\end{array}$ & $\begin{array}{r}.25 \\
2.20\end{array}$ \\
\hline Reodkapje (Pf.) .. & 1 & 1.00 & .90 & .80 & .70 & .60 & .50 & 10 & .90 \\
\hline $\begin{array}{l}\text { Rose Ash (D) } \\
\text { Ashes of Roses, Vigorous ... }\end{array}$ & $\begin{array}{r}10 \\
100\end{array}$ & $\begin{array}{r}.50 \\
4.00\end{array}$ & $\begin{array}{r}.40 \\
3.20\end{array}$ & $\begin{array}{r}.35 \\
2.50\end{array}$ & $\begin{array}{r}.25 \\
2.00\end{array}$ & $\begin{array}{r}.15 \\
1.00\end{array}$ & $\begin{array}{l}.10 \\
.80\end{array}$ & $\begin{array}{r}100 \\
1000\end{array}$ & $\begin{array}{l}.10 \\
.80\end{array}$ \\
\hline Rosemary (Bales) …............. & 10 & 1.75 & 1.50 & 1.25 & 1.00 & .75 & .50 & 100 & 1.25 \\
\hline Ruth Taylor (D) & 1 & 2.00 & 1.50 & 1.00 & & & & & \\
\hline Santa Barbara (Br.) & 1 & 3.50 & 3.00 & 2.50 & 2.00 & 1.50 & 1.00 & 10 & 2.50 \\
\hline Seabrook Gem (R.) & 10 & 1.00 & .85 & .70 & .55 & .40 & .25 & 100 & .20 \\
\hline Selma (Kemp) & 10 & 1.75 & 1.50 & 1.25 & 1.00 & & & 100 & .95 \\
\hline Bill) ... & 1 & .50 & .45 & .40 & .35 & .30 & .25 & 10 & .50 \\
\hline Sharon Rose (C) & 10 & 1.25 & 1.15 & 1.00 & .85 & .70 & .50 & 100 & .75 \\
\hline Shasta (Kir.) & 1 & .45 & .40 & .35 & $.30^{\prime}$ & .25 & .20 & 10 & .35 \\
\hline Sheila (C) & 19) & .65 & .55 & .45 & .35 & .25 & .15 & 100 & .25 \\
\hline Souvenir (Eu.) & i0 & & & & .30 & .25 & .15 & 100 & .10 \\
\hline Dee & 100 & & & & 2.0 & 1.50 & 1.00 & 1000 & .80 \\
\hline Stalwart (Kemp) & 10 & .75 & .65 & .50 & .40 & .30 & .20 & 100 & .25 \\
\hline Tall vigor & 100 & 6.50 & 5.25 & 4.00 & 3.00 & 2.00 & 1.00 & 1000 & 1.50 \\
\hline Star of the Sea (E) ... & 1 & 3.50 & 3.00 & 2.50 & 2.00 & 1.50 & 1.00 & 10 & 2.00 \\
\hline Sulphur Frills (K) & 10 & 2.00 & 1.75 & 1.50 & 1.25 & 1.00 & .75 & 100 & 1.00 \\
\hline 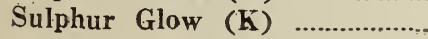 & 10 & .60 & .50 & .40 & .30 & & & 100 & .20 \\
\hline
\end{tabular}




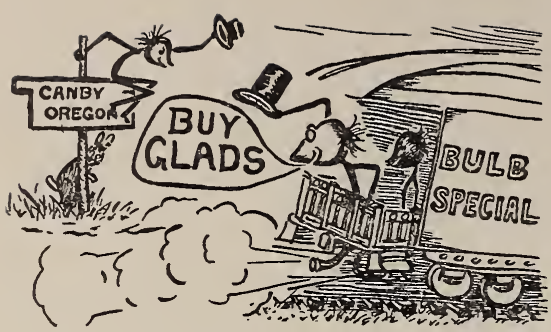

TERMS: Cash, or $25 \%$ down, and balance c. o. d.

If not otherwise listed, you may have 1000 at 9 times $100 ; 5000$ at $41 / 2$ times $1000 ; 250$ at $1 / 4$ of 1000 rate. Prices are arranged so that small orders are retail and large orders wholesale. For discounts and special offers see next to last page. You may also write us for special quotations in quantity.

We pay transportation on orders over $\$ 3.00$.

Bulbs
Per

Sunnymede (Fis.) Buff Orange 10)

Sunset Glow (G)

Superba (C)

Sweetheart (Kemp) Cream

Sword of Mahomet (E)

Tamalpais (D)

The Aristocrat (Bill) Saffron Pink

The Orchid (Spr)

The President (K)

Thos. A. Edison (D)

Begonia Rose

Thos. A. Edison (K)

Tiger ( $K$ )

Titan (Australia)

Trilby (Pr)

Troubadour (Pf.)

Tycko Zang (A)

Large Salmon Pink

Tyrian Beauty (K)

Uhlan Chief (K)

Vanity (Bill)

Van Wert (K)

Veilchenblau (Eu.)

Veiled Brilliance (A)

Vesta Tilly (Eu.) …................ 10

White. Stands heat ............... 100

Virginia M. Fisher (D) ......... 10

Martha Wash. Geranium .... 100

Vivian Mae Wilson (W) ........ 1

W. A. Sisson (D) ................... 1

Lilac-Tinted White ............ 10

West Virginia (R) ................. 10

White Cloud (E) ..................... 1

White Dove (M) ...................... 10

White Orchid (Bill)

White Premier ........................ 1

Wm. G. Badger (M) .............. 10

Fine tall Pink ....................... 100

Wm. Kent (D) ....................... 10

Ruffled Cream ...................... 100

White Wonder (Kemp) .......... 10

Tall White ............................. 100

Wotan (D)
No. 1 No. 2 No. 3 No. 4

$1 \frac{1 / 2}{\text { up }} 1 \frac{1 / 4}{1 / 1 / 2} \quad 1-1 \frac{1 / 4}{3 / 4}-1$

1.25

10.00

.75

.80

7.00

1.00

.65

1.40

12.00

$$
1.00
$$

8.50

.80

.65

.65

5.50

.85

.55

1.20

10.50

1.25

1.10

1.25

10.00

15.00

.95

8.75

12.00

.45

.50

.45

1.00

1.50

1.35

12.00

.60

.80

1.00

1.25

.90

1.15

1.00

9.00

2.50

2.00

15.00

2.00

1.00

2.00

2.00

.45

.75

6.50

2.00

15.00

.55

4.25

1.00
.40

1.25

1.00

9.00

.50

.70

.85

1.10

.75

1.00

.85

7.50

2.25

1.75

12.50

1.75

.85

1.75

1.75

.40

.60

5.00

1.65

12.50

.45

3.85

.85
5.50

.55

1.15

.50

4.00

.70

.45

1.00

9.00

.60

.90

.85

7.50

10.00

.40

.35

.80

1.15

.75

6.25

.40

.55

.70

1.00

.60

.85

.70

6.00

2.00

1.50

10.25

1.50

.70

1.50

1.50

.35

.45

3.25

1.25

10.25

.40

3.20
.45

.90

.40

3.00

.55

.35

.85

7.50

.50

.75

.75

6.25

.35

.30

.70

1.00

.50

4.25

.30

.40

.55

.85

.50

.70

.25

2.00

.55

4.50

1.25

8.75

1.25

.55

1.25

1.25

.30

.25

1.85

1.00

8.75

.30

2.40

.60

No. 5 No. 6 $1 / 2-3 / 4$

.50

$\begin{array}{ccr}.50 & .35 & 100 \\ 4.00 & 2.50 & 1000\end{array}$

1/2-Less

Bulblets

Per

.65

.30

2.00

.40

.25

.70

6.00

.40

.60

.60

5.00

.30

.25

.60

.90

.35

3.00

.25

.30

.70

.40

.55

.15

1.00

.40

3.00

1000

.25

1.75

1.15

$\begin{array}{rr}.40 & 100 \\ 20 & 100\end{array}$

.40

$\begin{array}{rr}1.00 & 1000\end{array}$

.25

$.25 \quad 10$

1.25

.15

.55

4.50

10

.50

.30

100
1000

.45

10

8.25

.50 


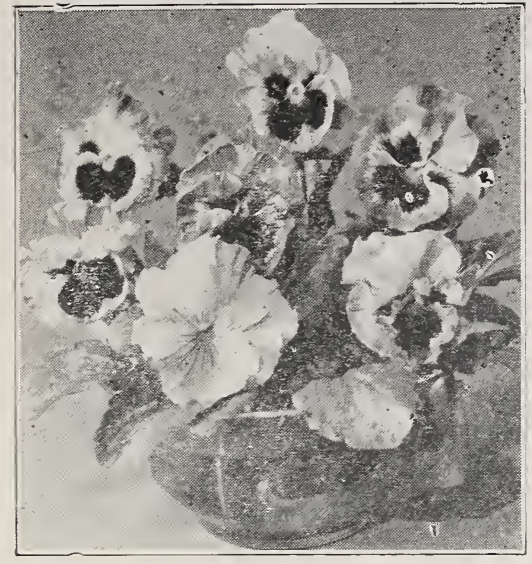

\section{Oregon Giants}

A wcncerful strain of very large, ruffled pansies criginated $b y$ Mrs. Merton G. Ellis.

Finest Florists' Mixture

600 Seeds …............................... $\$ 1.00$

Trial Pachet of 175 Sceds ...................... 35

WHAT OTHERS THINK OF OREGSN GIANTS

"The Oregon Giants make other kind- look like a joke."-11. B. Thempson, Tuscumbia, Ala.

"We would not waste our time yroving aiher varieties. "-Mrs. J. M. Whitehead, Ponena, Crlif.

"Why should we plant the tiny ones when there are such beautes to be had." - lirs. E. i. i.....t, 1535 Central. Alandeclat. Calif.

Ycur fiower-loving friend w'ul appreciate a $p$ cket of $\mathrm{OREGON}$ GLANT PANSY SEED.

Wrexham Delphinium

Seed $\$ 1.00$ Pcr Pit.

Columbine or

Acquilegias …...... .50 Per Pkt.

Double Poppies

.15 Per Pkt.

All orders are suljzct to prior sal a and to prices on list from which ordered regardless of future rise or fall in price.

\section{$\therefore \quad \therefore$ \\ New Dahlias}

CANBY QUEEN-(Mrs. M. G. E. 1927) Of the peony type, but very double and sclli.m shows the center. Very large, often 11 in.he across and stands up well on strong stems. Very delicate lavender pink, with fine yellow line through each petal. ......................... $\$ 4.90$

ROSE GLOW-(Mrs. M. G. E. 1923) Manmoth clear bright rose exhibition variety, strung growing, very tall, and is not afraid to lonk the world in the face. Must be aeen to br appreciated.

$x=(40$

\section{GLAD SPECIALS}

Set E1: One bulb each of any six of the followin 3 in blooming size bulbs. $\$ 10$

Angel's Dream Ella V. Birney

Beatricia

Eloma

Gloriola

White Cloud

Red Roy

Red Marie

Pink Marie

Capt. Courageous

Pride of Portland

Star of the Sea

Sword of Mahomes

Miss Canby

Early Phipps

Lady Lorene

Red Tornado

Rippling Waters

Set E2: One bulb each of all varieties in Set E1, in blooming size bulbs

$\$ 20$

Set E3: Five bu'blets each of any six varieties in Set $\mathrm{E} 1$ $\$ 10$

Set E1: Five bulblets each of all varieties in set $\mathrm{E} 1$

Set E5: 1 b'oom:nr size bu'b and ten bulblets of Lady :IacBath, Ernestine, Mountain Girl, Dorothy Wood and Bengal Beauty $\$ 10$

\section{DOROTHY WOOD SETS}

(a) 1 No. 1 \& 12 Blts.

(b) 1 No. 3 \& 20 Blts.

(c) 1 No. 4, 1 No. 6, \& 10 Blts, ...5.00

\section{$\therefore \quad \therefore$}

"l know of no other orig nator-unless it be Joe Coleman-who has given such a high percentage of teal worthwhile varieties. Yours are uniformly, really worthy." Thus Gale W. Thacker writes us. He is widely recugnized as an authority on the new, rare and unusual cilads of merit.

Disccunts-Cn all cash orders you may select extra Bulbs ard Bulblets as follows: $5 \%$ extra on orders from $\$ 5.00$ to $\$ 25.00 ; 10 \%$ on $\$ 25.00$ to $\$ 5000 ; 15 \%$ on $\$ 50.00$ to $\$ 100.00 ; 20 \%$ cn $\$ 150.20$ or more.

\section{파표. \\ Regal Lilies}

Regal Lily Seed: Per packet, $\$ 1.00$. Per ounce, $\$ 2.50$. Per pound, $\$ 35.00$.

\section{REGAL LILY BULBS \\ (Fall delivery only).}

$\begin{array}{lccc}\text { Cir. in inches } & \text { Per } 10 & \text { Per } 100 & \text { Per } 1090 \\ 8 \text { to } 9 & \$ 9.00 & \$ 60.00 & \$ 500 \text { c0 } \\ 7 \text { to } 8 & 6.50 & 50.00 & 401.100 \\ 6 \text { to } 7 & 4.50 & 40.00 & 30000 \\ 5 \text { to } 6 & 3.50 & 30.00 & 250.00 \\ 4 \text { to } 5 & 2.75 & 25.00 & 2: \text { s. }^{2} 0 \\ 3 \text { to } 4 & 1.75 & 15.00 & 125.00\end{array}$


A prominent Washington grower, who has acted as Judge at Glad Shows, writes us, "I think jroijably that table of yours would have stirred up about as much excitement as they had at all the Eastern shows put to. gether, had it been shown at one of their shows. You had more exceptional stuff on that table than I've ever seen before in the same space. They were wonderful." $\mathrm{He}$ refers to our recent exhibit at the Northwest Regional Glad Show where we took Grand-Sweepstakes Gold Cup for most points, Sweepstakes Silver Cily for best spike, First for Best Exhibit, First and Second for best new seedling, etc. About a third of our exls:bit cunsisted of our own uriginations.

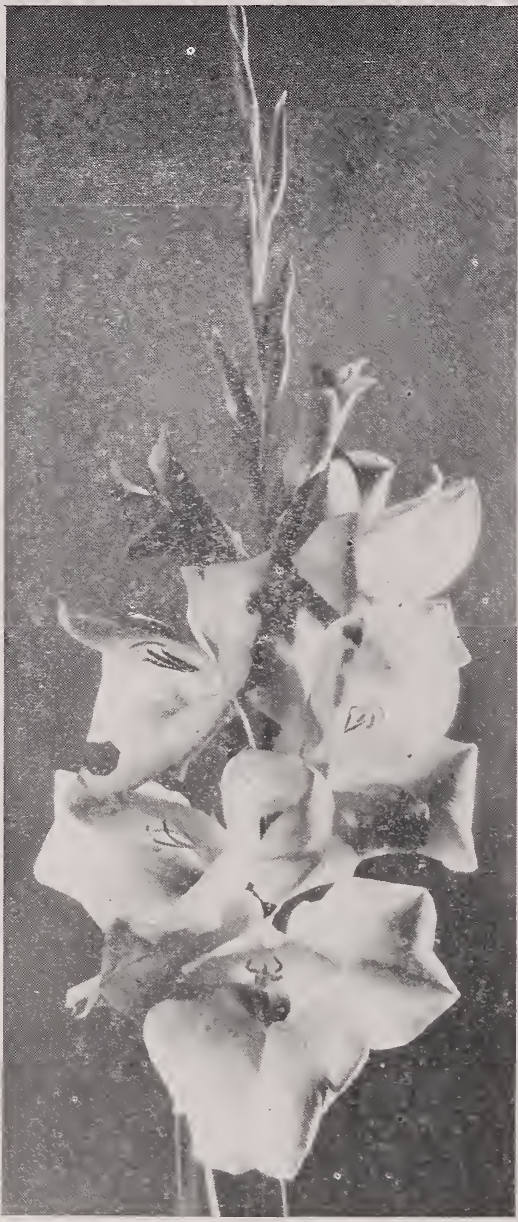

"Ella V. Birney"
"Early Phipps"

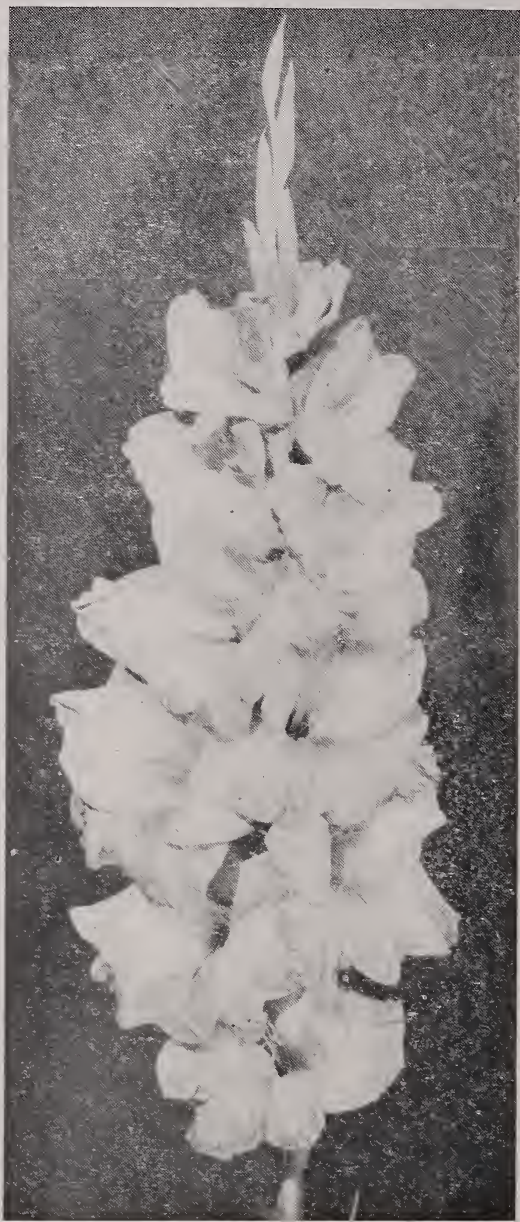

Prices are for cash. All orders are received subject to prior sale, and are shipped either postpaid or express prepaid. Bulbs may be shipped by express at any time during the winter. The express company insures iagainst freezing. If frozen in transit, please notify your express agent at once so that he may examine the bulbs, and also notify us at once so that we may file claim. Fy express, we guarantee safe delivery.

\section{Merton G. Ellis Bulb Gardens CANBY, OREGON}

